\title{
Vergleichende Untersuchungen der zur Bestimmung des Glycyrrhizins in der Sulsholzwurzel und im Succus Liquiritiae vorgeschlagenen Methoden.
}

\author{
Von A r min $\mathrm{I}_{\text {i }} \mathrm{nz}$.
}

(Preisarbeit der Hagen-Bucholz-Stiftung 1913/14.)

Seit sehr langer Zeit schon hat man sich in einer großen Zahl von Arbeiten und Veröffentlichungen mit den Bestandteilen der Süßholzwurzel und der Lakritzen beschäftigt. Schon um das Jahr 1800 herum machten Pfaff, Herm bstäd $\mathrm{t}$, S chwartze Angaben über die Zusammensetzung der Süßholzwurzel und des Extraktes, gaben auch schon charakteristische Fällungen eines Aufgusses mit verschiedenen Reagentien an. In den darauf folgenden Jahrzehnten nimmt die diesen Gegenstand berührende Literatur einen bedeutenden Umfang an. Alle diese Arbeiten bringen jedoch bis etwa 1880 fast nur Vorschläge zur Gewinnung des der Wurzel und dem Iakritzen eigentümlichen Stoffes, ohne auf eine quantitative Bestimmung desselben Wert zu legen. Wenn auch aus diesem Grunde obige Arbeiten keinen direkten Zusammenhang mit den zur Bestimmung des Glycyrrhizins benutzten Methoden haben, so glaubte ich doch einen Ueberblick über dieselben geben zu müssen. Einesteils, weil naturgemäß zwischen den ersten Versuchen der Isolierung eines Körpers und dann seiner quantitativen Bestimmung eine gewisse Abhängigkeit vorhanden ist, dann aber auch, weil eine solche Zusammenstellung unter Berücksichtigung aller erschienenen Arbeiten noch nicht versucht worden ist ${ }^{1}$ ). Die kurzen Uebersichten, die den Arbeiten von $T \mathrm{sch}$ i $\mathbf{r} \mathrm{ch}, \mathrm{R}$ a se $\mathrm{nak}$, Ced e rberg, Ga u chma n n vorangehen, sind unvollständig und zum Teil auch fehlerhaft.

1) Der Inhalt dieser Publikationen ist in der Originalarbeit in entsprechender Weise mitgeteilt. An dieser Stelle mag es jedoch genügen, das Wesentliche der Arbeiten, welche die quantitative Prüfung des Lakritzen und der Süßholzwurzel betreffen, nach den Angaben des Verfassers mitzuteilen, im übrigen aber auf das Literaturverzeichnis in Anlage $\mathbf{A} \mathbf{z u}$ verweisen.

Redaktion. 
In der Anlage $\mathrm{A}$ ist ein Verzeichnis aller der Arbeiten beigefügt, welche sich mit den Inhaltstoffen der Süßholzwurzel und der Lakritzen, mit ihrer Chemie und ihrer quantitativen Bestimmung beschäftigen. Diese Aufstellung kann für sich in Anspruch nehmen, alle hierher gehörenden wichtigeren veröffentlichten Arbeiten berücksichtigt zu haben. Auch ein solches Verzeichnis ist noch nicht aufgestellt worden. Die Uebersichten in den älteren Werken, wie Flü ckiger: Pharmakognosie, $\mathbf{H}$ u s e mann: Pflanzenstoffe, dann aber auch in Ts o h i $\mathrm{r}$ h: Handbuch der Pharmakognosie, Dragend orff: Die Heilpflanze, We hmer: Pflanzenstoffe, sind bei weitem nicht vollständig. Da auch der Jahresbericht der Pharmazie nicht alle Arbeiten gebracht hat, war ein solches Verzeichnis aufzustellen nur möglich nach Durchsicht aller in Betracht kommenden Zeitschriften.

Durch gütige Vermittelung" einiger Herren wurde es mir ermöglicht, fast sämtliche Arbeiten im Original einsehen zu können. Auch der Benutzung des augenblicklich im Druck befindlichen Zeitschriftenkataloges der Auskunftsstelle deutscher Bibliotheken, ebenso wie der Bibliothek des Deutschen Apotheker-Vereins und des Reichsgesundheitsamtes verdankte ich die Möglichkeit, fast alle in ausländischen Zeitschriften erschienenen Arbeiten im Original bearbeiten zu können. Bei den Veröffentlichungen, die ich nicht im Original einsehen konnte, habe ich die mir zugängliche und von mir benutzte Arbeit, die einen Auszug aus dem Original brachte, angegeben.

\section{Die quantitative Glycyrrhizinbestimmung in den Lakritzen.}

Der Gedanke, den Gehalt an Glycyrrhizin quantitativ festzustellen und ihn zur Bewertung der Lakritzen zu benutzen, ist auf $R$ u m $p$ zurückzuführen. Wie ich schon erwähnt habe, stellte er im Jahre 1855 den Satz auf: Es läßt sich der Wert der Lakritzen ebenso wie beim Opium an dem Morphingehalt durch den Gehalt an Glycyrrhizin am besten bestimmen. Dieser Satz ist nicht unwidersprochen geblieben. Kurze Zeit später erklärt $\mathrm{H}$ a g e $\mathbf{r}$ das Gegenteil. Trotzdem aber stellt er die Mindestforderung von 10 a. $\mathbf{H}$. Glycyrrhizin in den Lakritzen auf. Die nächsten Jahrzehnte haben eine große Zahl von Vorschlägen gebracht, das Glycyrrhizin der Menge nach festzustellen, woraus zu schließen ist, daß dieser Bestimmung ein Wert für die Feststellung der Güte einer Lakritzenart zugeschrieben werden muß. In den letzten Jahren aber hat man auch der Zuckerbestimmung erhöhte Aufmerksamkeit gewidmet. Und dies mit Recht. Außer den selbstverständlichen Bestimmungen 
des Lösliohen und Unlöslichen und der Asche ist die Glyoyrrhizinsăure- und Zuckerbestimmung zu einer erschöpfenden Bewertung unumgänglich nötig. Durch eine Bestimmung der Glycyrrhizinsäure allein könnte man zum Beispiel die häufig vorkommende Verfälschung eines viel Glycyrrhizinsäure enthaltenden Succus mit Zucker nicht nachweisen. Deshalb haben auch sehr viele der neuen Bearbeiter dieser Frage eine solche Bestimmung aufgenommen. Ich will hier nur nennen $\mathrm{Houseman,Telle,Pary,}$ Tschirch usw.

Ein der Zeit nach geordnetes Verzeichnis der bis jetzt veröffentlichten Glycyrrhizinsäurebestimmungen habe ich am Schluß der Arbeit in der Anlage B zusammengestellt.

Eine Anzahl von Prüfungsvorschlägen, besonders von solchen, die in Sammelwerken aufgenommen wurden, sind zum Teil anderen Arbeiten entnommen und erscheinen dann, teilweise unter anderen Namen, als selbständige Bestimmung. So fand ich unter dem Namen Prollius in Hager-Fis cher-Hartwich's: Handbuch der pharmazeutischen Praxis 1896 den Prüfungsvorschlag Diehl's. Hager's Handbuch hat im Ergänzungsband die Haffner'sche Prüfung. König: Nahrungs-und Genußmittel I., S. 1065 hat die Prüfung nach Kremel übernommen.

In das Verzeichnis der Anlage B habe ich nur selbständige Prüfungen aufgenommen.

Aus der großen Zahl der quantitativen Arbeiten kann man ersehen, zwischen welchen Werten der Glycyrrhizingehalt der Lakritzen sohwankt. G 1 ü $\mathrm{cks} \mathbf{m}$ a $\mathbf{n} \mathbf{n}$ hat in einer Lakritzensorte gar keine Glycyrrhizinsäure gefunden, andere wollen in verschiedenen Marken bis fast 30 a. H. gefunden haben. Aber nicht allein in den verschiedenen Sorten, sondern auch in derselben zu verschiedenen Zeiten sind große Schwankungen im Glycyrrhizinsäuregehalt festgestellt worden. Eine Tatsache, die ihre Erklärung wohl in den höchst primitiven Darstellungsverfahren findet, wie sie zum Teil noch angewendet werden. Aus diesen Gründen rechtfertigt sich die oft ausgesprochene Forderung, eine untere Grenze des Glyoyrrhizingehaltes durch das Arzneibuch festzulegen oder aber die Selbstbereitung durch eine Arzneibuchvorschrift anzuregen.

Aus den sehr wenigen, bisher vorliegenden Vergleichen verschiedener Bestimmungen aber ist wieder zu ersehen, daß man nach den zahlreichen Prüfungsvorschlägen voneinander ganz abweichende Werte erhält. Ich möchte hier zum Beispiel E $\mathbf{r}$ i k s on anführen, die nach ihrer Bestimmung 16,5 a. $\mathrm{H}$., nach der $\mathrm{Ced}$ e $\mathrm{rb}$ er g's 
14,3 a. H. Glycyrrhizinsäure bei Verwendung des gleichen Succus erhält. G 1 ӥ c k s m a n n will aus dem ammoniakalischen Auszug 8, aus dem wässerigen nur 2 a. H. Ammonglycyrrhinat erhalten haben. $\mathrm{H}$ a f $\mathrm{n}$ er hat noch der Arbeitsweise von $\mathrm{Helf}$ en berg 4,3, von $\mathrm{Kremel} 3,1$, von $\mathrm{Diehl} 6,4$ a. $\mathrm{H}$. bei Verwendung gleicher Lakritze gefunden. Interessant sind auch die Tabellen von H a f $\mathrm{ner}$, in denen er einen Zusammenhang zwischen Auszugsflüssigkeit, Reinigungsmittel und dem Reinheitsgrad der erhaltenen, zur Wägung gebrachten Säure festzustellen glaubt.

Diese wenigen vergleichenden Angaben genügen aber schon, uns zu dem Schluß zu berechtigen, daß die Frage der Glycyrrhizinsäurebestimmung noch ungeklärt ist. Diese Tatsache, verbunden mit der Notwendigkeit einer solchen Bestimmung, gibt der diesjährigen Arbeit der Stiftung einen hohen praktischen Wert.

Verschiedene, welche auf diesem Gebiet gearbeitet haben, haben die Beschäftigung mit dem Glycyrrhizin, sowohl in chemischanalytischer, als auch in quantitativer Hinsicht als "undankbar". bezeichnet. Es ist dies zu verstehen, besonders in letzterer Hinsicht. Ein reines Glycyrrhizin oder eine ebensolche Verbindung quantitativ zu erhalten ist meines Erachtens noch nicht möglich. Man erhält nur mit mehr oder minder großen Verlusten eine mehr oder weniger stark verunreinigte Säure oder deren Verbindung.

\section{Einleitung zu den quantitativen Bestimmungen.}

Bevor ich auf die von mir unternommene Nachprüfung der einzelnen Bestimmungsvorschläge eingehe und deren Ergebnisse mitteile, möchte ich an dieser Stelle einige grundlegende Fragen erörtern, die für alle vorgeschlagenen Prüfungen von Wichtigkeit beziehungsweise einem größeren Teil derselben gemeinsam sind. Tch glaube, hierdurch unnötige Wiederholungen vermeiden zu können.

Es wäre hier zu erörtern der Einfluß der Flüssigkeit, welche als Lösungsmittel des Succus benutzt werden soll. Weiter wäre Rücksicht zu nehmen auf die zur Fällung benutzte Säure. Es müßte dann die Löslichkeit der Glycyrrhizinsäure im Fällungsmittel und in Wasser und die durch sie veranlaßten Verluste untersucht, und endlich die Reinheit der zur Wägung gebrachten Substanz berücksichtigt werden. Auf die vielen Fragen, die nur einzelne Bestimmungen berühren, möchte ich bei den betreffenden Prüfungen selbst eingehen. 
1. Diezum Auflösendes Succus benutzte Flüssigkeit.

Die ersten Vorschläge laufen naturgemäß darauf hinaus, den Succus in Wasser zu lösen. Es zeigte sich aber, daß diese Lösung außerordentlich schwer filtriert. Um diesem Uebelstand abzuhelfen, schlug im Jahre $1883 \mathrm{D}$ i e h l vor, nach der Lösung in Wasser eine gleiche Menge Spiritus hinzuzusetzen und erst dam, nach dem Absetzen, zu filtrieren. Es ist interessant, da $\beta \mathrm{D}$ i e h 1 diesen Vorschlag ausdrücklich mit der dadurch erzielten leichteren Filtration begründete. Er erreichte aber damit nicht allein diesen praktischen Zweck, sondern erzielte auch eine größere Reinheit der später ausgeschiedenen Säure. Durch den Alkoholzusatz werden in reichlicher Menge die im Succus vorhandenen Gummi- und Schleimstoffe gefällt, die sonst ins Filtrat übergegangen wären. Bei dem nun folgenden Ausfällen erhält man naturgemäß auch eine reinere Glycyrrhizinsäure. Auf jeden Fall ist der Spirituszusatz zum wässerigen Auszug als Fortschritt zu bezeichnen, durch den Verluste an Glycyrrhizinsäure im allgemeinen nicht entstehen können. Unter diesen Umständen ist es verwunderlich, daß nach $D$ i e $h l$, und auch noch in der letzten Zeit, Bestimmungen veröffentlicht wurden, die keinen Alkohol verwenden. Letztere sind ohne weiteres denen mit Alkoholzusatz unterlegen.

Der erste, der den ammoniakalisehen Succusauszug vorschlug, war, wie es scheint, im Jahre $1855 \mathrm{R} \mathrm{u} \mathrm{m} \mathrm{p}$. Wie ich schon in der Einleitung erwähnte, schloß er aus der Tatsache, daß das in Wasser Unlösliche noch eine gewisse Menge an Ammoniak abgab, anf die Anwesenheit eines in Wasser und eines nur im Ammoniak löslichen Glycyrrhizins. Auch S c h r öd e r stellt 1883 in einer Aufstellung dem löslichen ausdrücklich das unlösliche Glycyrrhizin gegenüber.

Ich halte diesen ammoniakalischen Auszug nicht für richtig.

Im einleitenden Teil habe ich darauf hingewiesen, daß T s ch ir ch das ,Glycyrrhizin" der Wurzel als eine Kalium- und Calciumverbindung der Glycyrrhizinsäure ansieht. Er gelangte zu dieser Ansicht auf Grund des folgenden Versuches: Ein gesättigter wässeriger Auszug der Wurzel wurde mit der gleichen Menge Weingeist versetzt, filtriert, und dann die dreifache Menge absoluten Alkohols hinzugefügt. Dadurch schieden sich die Glycyrrhizinverbindungen ab. Der Niederschlag wurde abfiltriert, in Eisessig gelöst und durch Umkrystallisation gereinigt. T s $\mathrm{ch}$ ir $\mathrm{ch}$ erhielt zwei Arten von Krystallen, die bei der qualitativen Analyse die Anwesenheit von Kalium und Calcium zeigten. 
Die von Flü ckiger 1867 aufgestellte Behauptung, das "Glycyrrhizin" sei das Ammonsalz der Säure, ist von vielen, darunter von $\mathrm{S}$ e s t i n i bezweifelt worden. T s c $\mathrm{h}$ ir $\mathrm{c} \mathrm{h}$ glaubt diese Ansicht endgültig widerlegt zu haben, da er bei der oben erhaltenen Fällung keine Ammonverbindung fand. Die an einer Stelle veröffentlichte Ansicht, es handle sich um ein Magnesiumsalz der Säure, ist an und für sich nicht unwahrscheinlich. Ihr ist bis jetzt nicht widersprochen worden. Außer der gebundenen Säure soll die Wurzel nach den übereinstimmenden Angaben der Literatur auch noch ganz geringe Mengen freier Glycyrrhizinsäure enthalten.

Bei der zum Teil noch sehr primitiven Darstellung der Lakritzen (siehe T s c h i r c h's Handbuch und A n s el m in o-Gilg: Kommentar) wird die Wurzel mit Wasser ausgekocht. Die geringen Mengen freier Säure werden dabei sicherlich durch die Bestandteile des Brunnenwassers oder auch durch sonstige Salze der Wurzel gebunden, so daß man also als feststehend ansehen kann, daß sich die Glycyrrhizinsäure in den Lakritzen nur in gebundener Form vorfindet. Ohne die Frage, welche Verbindungen im Succus vorkommen, entscheiden zu wollen, will ich hier nur mit der Möglichkeit rechnen, daß es sich in den Lakritzen um eine Kalium-, Calcium-, Magnesium- und Ammoniumverbindung handeln kann. Die Kalium- und Ammoniumverbindung ist in Wasser sehr leicht löslich. Ueber die Magnesiumverbindung verlautet in der Literatur nichts, sie scheint noch nicht dargestellt worden zu sein. Es ist aber nach der Natur der Magnesiumsalze anzunehmen, daß das Glycyrrhinat leicht löslich ist. Von dem Calciumsalz berichtet $\mathrm{Sestin} \mathrm{i}$, daß es in Wasser schwer löslich sei. Berücksichtigt man aber, daß bei den verschiedenen Bestimmungen nur ganz geringe Mengen des Calciumsalzes zu lösen sind, so wird man annehmen können, daB das glyoyrrhizinsaure Calcium bei Verwendung angebrachter Mengen Wassers in jedem praktischen Falle einer Bestimmung gelöst wird.

Alle als "Glycyrrhizin" in den Lakritzen möglichorweise enthaltenen Verbindungen sind also für unsere praktischen Prüfungsverhältnisse wasserlöslich. Eine freie Säure, die durch Alkali löslich gemacht werden müßte, ist nicht vorhanden. Es erübrigt sich also, zu der Auszugsflüssigkeit Ammoniak hinzuzusetzen. Aber dieser Zusatz ist nicht allein unnötig, sondern sogar unzweckmäßig, denn $\mathrm{H}$ aff $\mathrm{n}$ er hat nachgewiesen, daß das glycyrrhizinsaure Calcium in Ammoniak außerordentlich schwer löslich ist. Gegen einen solchen Auszug spricht aber noch eine andere wichtige Tatsache. Es steht fest, daß Ammoniak aus dem in Wasser unlöslichen Rückstand noch bedeutende Mengen herauslöst. Kann man nun die ge- 
samten Glyoyrrhizinsäure-Verbindungen durch Wasser herauslösen - und dieser Meinung bin ich, bei Anwendung natürlich nicht zu geringer Mengen Wassers - so wäre es falsch, durch Benutzung von Ammoniak noch mehr aus dem sonst Unlöslichen herauszuziehen, welches nicht Glycyrrhizinverbindungen enthält. Je mehr die filtrierte Auszugsflüssigkeit an ,Nichtglycyrrhizin“" enthält, um so unreiner muß natürlich auch die dann ausgefällte Glycyrrhizinsäure sein. Oder, positiv ausgesprochen: Die ausgefällte Glycyrrhizinsäure ist um so reiner, je größer der unlösliche Rückstand ist, vorausgesetzt natürlich, daß aus ihm die gesamte Glycyrrnizinverbindung herausgelöst worden ist. DaB ein ammoniakalischer Succusauszug noch bedeutend schwieriger filtriert als ein wässeriger, will ich hier nur erwähnt haben. Es ist nach obigem erklärlich, wenn ich bei den später zu erörternden Nachprüfungen bei Verwendung ammoniakalischer Austüge höhere Werte erhalten habe, als bei Benutzung reinen Wassers. Dieses Mehr tritt aber nur auf Kosten der Reinheit des zur Wägung Gebrachten. Aus allen diesen angeführten Gründen halte ich einen-ammoniakalischen Auszug für falsch.

Als dritte Auszugsflüssigkeit schlägt $H$ a $f$ f $n$ e $r$ ein Gemisch von Schwefelsäure und Alkohol vor. Alkohol selbst löst aus den Lakritzen fast nichts heraus, da die gesamten Glycyrrhizinverbindungen in Alkohol sehr schwer oder fast unlöslich sind. Durch den Schwefelsäurezusatz wird die Glycyrrhizinverbindung zersetzt, es bildet sich dabei die freie Glycyrrhizinsäure, die in dem Gemisch von Alkohol und Schwefelsäure leicht löslich ist. H a f $\mathrm{n}$ e $\mathrm{r}$ umgeht also, indem er die Glycyrrhizinsäure freimacht, die Frage nach der Löslichkeit der verschiedenen Verbindungen. Die Frage, ob durch diesen Aufschluß der Lakritzen auch das gesamte Glycyrrhizin herausgelöst wird, kann ich unbedingt bejahen. Daß die sämtlichen glycyrrhizinsauren Salze der Lakritzen durch die Schwefelsäure zersetzt werden, dürfte einem Zweifel nicht unterliegen, ebenso, $\mathrm{da} B$ die nun in Freiheit gesetzte Glycyrrhizinsäure sich in dem Schwefelsäure-Alkohol-Gemisch löst. Daher unterliegt der Vorschlag $\mathrm{H}$ a f $\mathrm{f} n$ er's kaum Einwänden in quantitativer Hinsicht.

Um festzustellen, ob sich in dem Trockenrückstand nach $\mathrm{H}$ a f $\mathrm{f} \mathrm{n}$ er noch in Ammoniak Lösliches befände, zog ich genau $5 \mathrm{~g}$ des Unlöslichen nach $\mathrm{H}$ a f f $\mathrm{n}$ e $\mathrm{r}$ mit ammoniakalischem Wasser aus. Auch nach dem dritten Auszug war die abgegossene Flüssigkeit noch tiefschwarz. Aus den vereinigten eingedampften Auszügen konnte ich noch $0,189 \mathrm{~g}$ Glycyrrhizin-Ammon nach der üblichen Bestimmungsweise feststellen. Da diese $5 \mathrm{~g}$ Rückstand ungefähr 
$10 \mathrm{~g}$ in Arbeit genommenen Succus entsprechen, wären in Rückstand nach $\mathrm{H}$ a f $\mathrm{n}$ e $\mathrm{r}$ noch fast 2 a. $\mathrm{H}$. Glycyrrhizin-Ammon nachgewiesen, die keine Glycyrrhizinsäure sind, aber bei ammoniakalischem Auszug des Succus, wahrscheinlich als Verunreinigung der Säure, als Glycyrrhizinsäure gewogen worden wären.

Meinè Ansicht über die verschiedenen vorgeschlagenen Auszugsflüssigkeiten möchte ich dahin aussprechen, daß sowohl der wässerige Auszug mit Alkoholzusatz, wie auch der Alkohol-Schwefelsäure-Auszug gute Ergebnisse gäben, daß diesen beiden Verfahren gegenüber aber der ammoniakalische Auszug nachsteht, weil er höhere Werte gibt, als tatsächlich vorhanden sind.

2. Die Löslichkeit ler Glycyr hizinsäure.

Versuche über die Löslichkeit der Glycyrrhizinsäure hat, soweit ich feststellen konnte, als erster $\mathrm{H}$ a f f n e r im Jahre 1899 angestellt. Daß solche Löslichkeitsuntersuchungen sehr wünschenswert seien, stellte schon $M$ a i s ch im Jahre 1884 in einem Nachwort zu dem Prüfungsvorschlag von $S \mathrm{ch} r$ ö d e r fest. H a f f n er schüttelte Glycyrrhizinsäure im Ueberschuß mit Wasser und. stellte dann durch Eindampfen das Löslichkeitsverhältnis fest. Er fand. die Zahlen $1: 60$, was also 1,67 a. $H$. gleichkommen würde.

Er betont ausdrücklich, daß er diesen Versuch nicht mit reiner Säure angestellt habe, mit der Begründung, daß eine solche bei der quantitativen Bestimmung doch nicht erhalten werde. Eingehende Löslichkeitsuntersuchungen stellt $\mathrm{Ca} \mathrm{p}$ in in seiner Dissertation an. Die vom Verfasser gezogenen Schlußfolgerungen sind aber zum Teil falsch. Ich möchte hier nur auf die Löslichkeitsuntersuchungen selbst eingehen. Die Benutzung derselben für seinen Prüfungsvorschlag werde ich besprechen, wenn ich die Glycyrrhizinsäurebestimmung nach $\mathrm{Ca} p$ in behandeln werde.

Wenn man zu einem Süßholzauszug oder einer Succus-, beziehungsweise Glyeinelösung Schwefelsäure zur Ausfällung hinzufügt, so bleibt die über der gefällten Glycyrrhizinsäure stehende Flüssigkeit gefärbt. Daraus folgert $\mathrm{C}$ a $\mathrm{p}$ i n wörtlich: ,Wenn nun aber die Gesamtheit der Glycyrrhizinsäure nach Hinzufügen der Schwefelsäure gefällt wäre, so ist es klar (évident), daß nach der Filtration die Flüssigkeit nicht die geringste Färbung zeigen würde." Diese Schlußfolgerung ist unbegreiflich. Sie hätte doch nur dann Sinn - und dann auch nur bedingungsweise - wenn die reine Glycyrrhizinsäure gefärbt, oder, besser, schwarz sein würde. Dies hat C'a p in geschrieben, trotzdem er die Arbeiten Tschirch's gelesen hat! Zur. Feststellung der Löslichkeit schüttelt $\mathrm{C}$ a $p$ i n 
$25 \mathrm{~g}$ Glycyrrhizinsäure (unreine, die er mit Wasser, Alkohol und Aether gewaschen hat) mit $200 \mathrm{ccm}$ Wasser und überläßt die Lösung während 24 Stunden sich selbst. Dann filtriert er und kühlt die klare Lösung im Eis-Kochsalzgemisch auf $0^{0}$ ab. Es zeigt sich von neuem eine Ausscheidung, die in einer Filtrieranlage, die eine Temperatur von $0^{0}$ gewährleistet, abfiltriert wird. Er trocknet dann $20 \mathrm{ccm}$ dieses Filtrats bis zum gleichbleibenden Gewicht und erhält $0,110 \mathrm{~g}$ Rückstand, was einem Gehalt von 0,55 a. $H$. entspräche (nicht 0,575, wie es infolge eines Druckfehlers in ( a p i n's Dissertation heißt). Unter denselben Versuchsbedingungen stellt er dann fest, daß der Löslichkeitsfaktor bei $15^{0} 0,575$ a. $H$. beträgt. Diese beiden Zahlen sind, wie schon oben bemerkt, vertauscht worden und zum Teil ist der Fehler auch in französische Zeitschriften übergegangen. Zum Beispiel in das Répertoire de Pharmacie III., 24, S. 14. Auf diesen Irrtum hin hat dann A ng u e t den Verfasser auf das Ungewöhnliche seiner Vorschrift hingewiesen, eine Temperatur von $0^{0}$ in seinem Prüfungsvorschlag vorzuschreiben, trotzdem die Löslichkeit nach eigenen Angaben ja bei $0^{\circ}$ höher sei als bei $15^{\circ}$. Ein Vergleich der Dissertation mit den gleichen Aufsätzen in dem Bulletin des Travaux de la Société pharmaceutique de Bordeaux zeigt sofort, daß es sioh hier nur um ein Versehen oder einen Druckfehler handeln kann. Die Schlußfolgerungen A n g u e t's sind daher also unberechtigt. Die Beobachtung C a p i n's, daß eine klare Lösung, aus welcher die Glycyrrhizinsäure herausgefällt worden ist, beim Abkühlen auf $0^{\circ}$ noch weitere Mengen von Säure ausscheidet, kann auch ich bestätigen. Ich habe sie besonders bei der später zu behandelnden Versuchsanordnung von Evans Sons gemacht. Durch diesen einfachen Versuch kann als festgestellt angesehen werden, daß die Glycyrrhizinsäure in Wasser bei $0^{\circ}$ weniger löslich ist, als bei $15^{0}$. Daraus ist die selbstverständliche Nutzanwendung auf die Prüfungsanordnung zu ziehen. Einen ähnlichen Weg zur Feststellung der bei der Glycyrrhizinbestimmung durch die Löslichkeit dẹr Säure in Wasser bedingten Verluste beschreitet $\mathrm{D}$ u r i e r.

Er löst soviel Glycyrrhizinsäure, wie er durch vorhergehende Versuche ausgefällt hatte, in $25 \mathrm{ccm}$ Wasser und, fällt mit Salzsäure. Dann filtriert er ab und wägt den Rückstand. Der Unterschied zwischen der verarbeiteten Menge Glycyrrhizinsäure und der zum Schluß gewogenen ist für ihn die Löslichkeitszahl in $25 \mathrm{ccm}$ Wasser. Auch hier liegt wieder ein Irrtum vor. D u r i e r stellt nicht die -Löslichkeit in Wasser, sondern in saurem Wasser fest. Diese beiden Löslichkeitszahlen sind aber ganz verschieden! Bei der Besprechung der D u r i e r'schen Arbeit werde ich näher darauf eingehen. 
Wir sehen, daß sich $\mathrm{H}$ a $\mathrm{f} \mathrm{f}$ er, D u rier und $\mathrm{Ca} p$ in mit der Löslichkeit der Glycyrrhizinsäure beschäftigt haben, und daß sie alle drei bei verschiedenen Versuchsbedingungen zu ganz verschiedenen Ergebnissen kommen. Unter diesen Umständen drängt sich die Frage auf: Haben die dahin gehenden Versuche Zweck? Theoretisch ist sie selbstverständlich zu bejahen, anders verhält es sich aber mit der praktischen Seite dieser Frage, was die Nutzanwendung für die vorliegende Arbeit anbetrifft. Es ist hier zuerst die Frage zu beantworten: soll das Löslichkeitsverhältnis der chemisch reinen oder der unreinen Säure untersucht werden. H a $f \mathrm{f}$ e $\mathrm{r}$ begründet seine Ansicht dahin : chemisch reine Säure kommt praktisch nicht in Frage, folglich benutzte ich zur Löslichkeitsbestimmung die unreine Säure. Ich bin derselben Meinung. Die Glycyrrhizinsäure einigermaßen rein und außerdem quantitativ zur Wägung zu bringen, ist bis jetzt niemandem gelungen und wird meines Erachtens auch nie gelingen. Deshalb halte ich es praktisch für überflüssig, feststellen zu wollen, inwieweit sich die reine Säure in Wasser löst, wenn man doch nur immer mit unreiner arbeiten wird. Man wägt immer "Glycyrrhizinsäure + Verunreinigung". Aus diesem Gemisch kann sich in Wasser sowohl die reine Säure, oder die Verunreinigung als auch Teile beider lösen. Da man nun die verunreinigte Säure zur Wägung bringt, wäre es falsch, nur das Löslichkeitsverhältnis des einen Teils der Summe "Glycyrrhizinsäure" + Verunreinigung zu berücksichtigen, das des anderen aber zu vernachlässigen. Aus diesem Gedankengang heraus müßte man also die Löslichkeit der unreinen Säure feststellen. Aber hier stehen der Ausführung mannigfache Sohwierigkeiten entgegen.

$\mathrm{H}$ a f $\mathrm{f}$ e r, C a p in, D u rier arbeiten jeder für sich mit ganz verschiedenen Stoffen. Es müßte also auch der Grad der Reinheit beziehungsweise Unreinheit berücksichtigt werden. Es wäre falsch von dem Ergebnis des an einer Säure angestellten Versuches auf alle anderen zu schließen. Wollte man genau arbeiten und die gefundene Zahl zu Korrekturzwecken der bei einer Glycyrrhizinbestimmung gefundenen hinzufügen, so müßte man bei einer jeden Glycyrrhizinbestimmung eine besondere Löslichkeitsbestimmung ausführen. Wollte man dann aber genaue Wägungen ausführen, müßte man von der bei $100^{\circ}$ bis zum gleichbleibenden Gewicht getrookneten Säure ausgehen. Hierbei haben sich aber die physikalischen Eigenschaften derselben zweifellos verändert. Ich will hier nur als Beispiel an die Aenderung der Löslichkeit gegen Alkohol erinnern, wenn man die Glycyrrhizinsäure ohne Temperaturerhöhung trocknet. Daß zum Beispiel durch Auswaschen der gefällten feuchten 
Säure dieselben Verluste entstehen würden, wie bei Verwendung vollkommen trockener, wäre widersinnig anzunehmen. Und trotzdem würde man doch diese Verhältnisse miteinander vergleichen.

Der von $\mathrm{Ca}$ p in und D u ri er gemachte Versuch, die Waschverluste, welche sich aus dem betreffenden Analysengang ergeben, durch Hinzufügen einer für alle Lakritzensorten geltenden Zahl zu dem durchVersuch festgestellten Glycyrrhizingehalt auszugleichen, ist in beiden Fällen nicht allein wegen der falschen Versuchsanordnung unbrauchbar, die zur Feststellung der Löslichkeitsverhältnisse führen sollte, sondern dem Vorschlag stehen auch grundsätzlich Bedenken gegenüber. Die von beiden gefundenen Zahlen beziehen sich naturgemäß nur auf gesättigte Lösungen, solche werden aber bei einer Glycyrrhizinbestimmung nie in Frage kommen. Es handelt sich ja bei dieser nur um das Auswaschen der gefälten Säure mit Wasser thd um die Löslichkeit derselben in der überstehenden angesäuerten Flüssigkeit. Daß hier also vollkommen verschiedene Verhältnisse vorliegen, die nicht miteinander verglichen werden können, liegt klar auf der Hand. Deshalb wird der Versuch Capin's und Durier's für die quantitative Bestimmung immer unbrauchbar bleiben. Gangbar wäre vielleicht ein anderer Weg, den ich unten erörtern will.

Zusammenfassend möchte ich also feststellen:

Die Löslichkeit der reinen Säure festzustellen, hat für die praktische Glycyrrhizinsäurebestimmung keinen Wert.

2. Eine Bestimmung der Löslichkeitsverhältnisse der unreinen Glycyrrhizinsäure kann nicht unter Bedingungen durchgeführt werden, die denen einer Glycyrrhizinsäurebestimmung gleichen oder ähneln.

Wenn auch die quantitativen Löslichkeitszahlen keinen Wert zu haben scheinen, so ist um so wichtiger die Feststellung folgender Tatsachen.

Die Glycyrrhizinsäure ist in Wasser in sehr geringem Maße löslich und zwar in Wasser von $0^{\circ}$ weniger als in solchem von $15^{\circ}$.

Die Glycyrrhizinsäure ist in angesäuertem Wasser ebenfalls etwas löslich, aber bedeutend weniger als in reinem Wasser.

3. Versuche, die durch die Löslichkeit der Glycyrrhizinsäure bedingten Verluste quantitativ festzustellen.

Die einzige Vorsohrift, die es bis jetzt versucht hat, die durch die Löslichkeit bedingten Verluste in jedem Falle einer Glycyrrhizinsäurebestimmung festzustellen und ebenfalls zur Wägung zu bringen, 
ist die Cornimboe u f's. Ich will sie an dieser Stelle behandeln, da Cornimboe u f nur die Bestimmung der Glycyrrhizinsäure im Glycyrrhizin. ammoniacal. behandelt, die ja dieser Arbeit fernliegt. Corminb o e u filtriert die mit Schwefelsäure gefällte Glycyrrhizinsäure $a b$ und löst sie in Ammoniak. Die überstehende abfiltrierte Flüssigkeit samt den Waschwässern dampft er fast zur Trockne ein, knetet den zähen schwarzen Rückstand mit je 10, 10 und $5 \mathrm{ccm}$ Wasser durch, filtriert die Waschwässer ab, löst die zurückbleibende zweite Menge Glyryrrhizinsäure in Ammoniak, vereinigt heide Ammonglycyrrhizinlösungen und trocknet sie bis zum gleichbleibenden Geqwicht. Ioh halte diese Versuchsanordnung nicht für einwandfrei.

Ich hatte des öfteren unabhängig von Cornimboe uf versucht, auf die gleiche Art zu der Möglichkeit zu gelangen, die Verluste an Glycyrrhizinsäure der Menge nach wenigstens annähernd festzustellen. Aber ich mußte regelmäßig die gleiche-Beobachtung machen. War das Eindampfen der Mutterlauge und der Waschwässer weiter fortgeschritten, so schied sich die Glycyrrhizinsäure zuerst in unansehnlichen braunen Flocken $a b$, die mit dem weiter fortschreitenden Eindampfen tiefschwarz wurden. Sie erwiesen sich als sehr schwer, zum Teil ganz unlöslich in Ammoniak. Zieht man in Erwägung, daß sich beim Eindampfen clas Wasser verflüchtigt, nicht aber die zur Fällung benutzte Schwefelsäure, daß sich also zum Schluß die Glycyrrhizinsäure im Gemisch mit einer sehr starken Schwefelsäure befindet, so findet man eine hinreichende Erklärung für diese Erscheinung. Es haben sich unter dem Einfluß der starken Säure Zersetzungserscheinungen eingestellt, zum Teil wird auch Verkohlung eingetreten sein, besonders dann, wenn man das Eindampfen nach C or n i m b o e u f's Vorschrift fast bis zur Trockne durchgeführt hat.

Beim Auskneten der eingedickten Säure mit $25 \mathrm{ccm}$ Wasser ergeben sich natürlich auch Verluste, aber solche sind ja bei der Glycyrrhizinbestimmung leider nicht auszuschließen.

Ich habe einen anderen Weg eingeschlagen, der meines Erachtens geeignet ist, eine ziemlich genaue Feststellung der Verluste an Glycyrrhizinsäure zu ermöglichen.

Die Mutterlange und die Waschwässer dampfte ich nach dem Sättigen der freien Säuren mit Ammoniak bis zur Sirupdicke ein. Diese gesättigte Lösung von Ammonglycyrrhinat und Ammonsulfat beziehungsweise -chlorid führte ich in einen schmalen Glaszylinder über, welcher eine Skala für halbe und ganze Kubikzentimeter aufwies. Die Lösung wurde dann soweit aufgefüllt, daß auf 
je ein Gramm des in Arbeit genommenen Succus $4 \mathrm{~g}$ Lösung kam. Dann fällte ich die Glycyrrhizinsäure mit Schwefelsäure aus, von der igh auf je ein Gramm Säure 10 Tropfen verwendete. Nach zwölfstündigem Stehen wurde durch ein kleines Faltenfilter von 5 ccm Durchmesser filtriert, die Säure auf dem Filter gesammelt: und mit Schwefelsäure $\left(2\right.$ a. H.) von $2^{\circ} \mathrm{C}$. ausgewaschen. Ich benutzte hierzu je nach der Menge des Niederschlages 5-10 com. Es wurde dann mit 5--10 ccm äthergesättigtem kaltem Wasser von $2^{\circ} \mathrm{C}$. nachgewaschen und der Rückstand im Vakuumexsikkator über Schwefelsäure getrocknet. Dann wird das Filter mit heißem 95 a. H. Alkohol erschöpft, die alkoholische Lösung eingedampft und als Glycyrrhizinsäure der Rückstand gewogen. Ich habe bei meinem Verfahren die Möglichkeit einer Zersetzung ausgeschlossen. Das Ammonglycyrrhinat ist so beständig, daß es ein Eindampfen selbst bis zur Trockne verträgt. Daß sich beim Fällen wieder Verluste durch Löslichkeit in der überstehenden Flüssigkeit einstellen, ist leider nicht zu vermeiden. Sie sind auch nur ganz gering. Auch das Auswaschen der Glycyrrhizinsäure bringt Verluste, die aber ebenfalls nur ganz gering sind. Ich glaube, daß ich bei genauer Befolgung obiger Versuchsanordnung quantitativ brauchbare Ergebnisse erhalten habe, die einen interessanten Aufschluß über die hei den einzelnen Bestimmungen erhaltenen Verluste geben.

\section{Die zum Ausfällen benützte Säure.}

Die Glycyrrhizinsäure ist eine schwache Säure, die sich durch die meisten anderen Säuren ausfällen läßt. Für die quantitative Glycyrrhizinsäurebestimmung ist es natürlich von Wert, zu wissen, welche Säure am geeignetsten zur Ausfällung erscheint. Bei den zu untersuchenden Prüfungsvorschlägen werden als Fällungsmittel benützt Schwefelsäure, Salzsäure und Alkohol absolutus. Im Verhältnis zueinander untersuchte ich die Wirkung von Schwefel-, Salz- und Borsäure, von Oxal-, Weinsäure und von Phosphor- und Ameisensäure als Fällungsmittel. Ich stellte mir eine Lösung dar, die, abfiltriert, das Lösliche aus dem Succus im Verhältnis $\mathbf{1}+\mathbf{9}$ enthielt. Von dieser Lösung wurden je $10 \mathrm{~g}$ in ein weites Reagenzglas überführt und dann abwechselnd mit 2,0 verdünnter Schwefelsäure, 3,0 verdünnter Salzsäure, 5,0 Phosphorsäure, 15,0 heißgesättigter Borsäurelösung, 15,0 Weinsäurelösung (5 a. H.), 3,0 Salpetersäure, 10,0 Ameisensäure hinzugefügt. Dann wurde der Inhalt eines jeden Glases auf 25,0 aufgefüllt. Vorher hatte ich mich an einem Kontrollversuch überzeugt, daß die von mir zur Ausfällung benützten 
Säuremengen zur Ausfällung gentigten. Naoh 24 stündigem Stehen und Abfiltrieren war bei diesen Kontrollversuchen auf erneuten Zusatz des gleichen Fällungsmittels Glycyrrhizinsäure nicht ausgeschieden worden. Nach 24 stündigem Stehen filtrierte ich die nach obiger Vorschrift behandelten Lösungen ab. Die Weinsäure hatte den Inhalt des Glases gelatiniert, sodaß man es umkehren konnte, ohne daß etwas herauslief. Sie kommt zur Ausfällung also nicht in Betracht, ebensowenig wie die Essigsäure, in der die Glycyrrhizinsäure leicht löslich ist. Die Farbe des Filtrats wie der gefällten Säure zeigte große Verschiedenheit. Das Filtrat der Borund Phosphorsäurefällung sah hellbraun aus, das der Schwefel-, Salz- und Salpetersäure mehr oder weniger dunkelbraun bis schwarz. Die gefällte Säure sah schwarz und unansehnlich aus, nur bei der Oxalsäurefällung hatte sie eine hellgraue Farbe, die wohl auf ausgefällte Calciumsalze zurückzuführen ist. $\mathrm{Zu}$ jedem der klaren Filtrate fügte ich dann noch $2 \mathrm{ccm}$ verdünnter Schwefelsäure. Nach zehnstündigem Stehen zeigte es sich, daß alle Lösungen mit Ausnahme der schwefel- und salzsauren erneut Glycyrrhizin ausgefällt hatten. Die Menge der Fällung war nur schwach bei der Phosphor-, Salpeterund Oxalsäure, stark aber bei der Bor- und Ameisensäure. Umsetzungen zwischen dem ersten Fällungsmittel und der geringen Menge verdünnter Schwefelsäure sind nicht zu befürchten, so daß diese nicht an der Fällung beteiligt sein können. $\mathrm{Zu}$ demselben Ergebnis bin ich durch einen zweiten Versuch gekommen. Ich stellte mir eine durch alkoholischen Auszug der getrockneten Säure gereinigte Glycyrrhizinsäure dar und schüttelte eine größere Menge als aufgenommen werden konnte mit Wasser. Je $20 \mathrm{ccm}$ des klaren Filtrats versetzte ich im weiten Reagenzglas mit je $2 \mathrm{ccm}$ der verschiedenen Säuren. Schwefel-, Salz- und Phosphorsäure gaben sofort eine Trübung, Oxal- und Salpetersäure erst nach einiger Zeit. Zuletzt gab auch Ameisensäure eine gelinde Trübung. Nach 24stündigem Stehen filtrierte ich ab, und es zeigte sich nach Zusatz von $2 \mathrm{ccm}$ Sohwefelsäure in der dadurch noch verdünnteren Glycyrrhizinlösung nach zehnstündigem Stehen bei allen Lösungen mit Ausnahme der salz- und schwefelsauren eine flockige Ausscheidung von Glycyrrhizinsäure. Aus diesen beiden einfachen Versuchen ist nur ein Schluß möglich: Schwefel- und Salzsäure reagieren schärfer als alle anderen Fällungsmittel. Um nun entscheiden zu können, welche von diesen beiden Säuren stärker wirkt, ging ich folgendermaßen vor.

Eine gesättigte Glycyrrhizinsäurelösung verdünnte ich einmal mit der doppelten, dann mit der dreifachen Menge Wasser und fugte 
zu je $10 \mathrm{ccm}$ dieser Lösungen $1 \mathrm{ccm}$ verdünnter Schwefelsäure und $1 \mathrm{com}$ verdünnter Salzsäure. Nach Verlauf einiger Stunden zeigten alle vier Reagenzgläser noch einen flockig-gelatinösen, leichten Niederschlag. Von einer neuen gesättigten Lösung - um dem Einwand einer möglichen Zersetzung entgegenzutreten - stellte jich mir eine Verdünnung etwa im Verhältnis $1: 21 / 2$ her und fügto wieder zu je $10 \mathrm{ccm}$ je $1 \mathrm{ccm}$ der beiden Säuren. Diesmal blieb die salzsaure Lösung auch noch nach 24 Stunden vollständig klar und durchsichtig, während die schwefelsaure Lösung auch jetzt noch eine sehr leichte, trotzdem aber unverkennbare Trübung aufwies. Ein Beweis, daß die Schwefelsäure zweifellos quantitativ besser ausfällt und die Glycyrrhizinsäure in schwefelsaurem Wasser schwerer löslich ist, als in salzsaurem. Auch die verschiedene Schnelligkeit, mit der die Fällung eintrat, spricht auf jeden Fall für die Schwefelsäure. Die Schwefelsäure ist also zweifellos die zum Ausfällen geeignetste Säure. Ich habe bei diesen Versuchen absichtlich jede quantitative Bestimmung mit Hilfe der chemischen Wege vermieden, da es sich ja hier nur um ganz geringe Mengen eines nicht einheitlichen Körpers handelt. Unter diesen Umständen könnte man Unterschieden in der dritten Dezimale kaum besondere Bedeutung beilegen. Ich glaube aber, daß für die praktischen Zwecke einer Glycyrrhizinbestimmung meine beiden Versuche genügend beweiskräftig sind. Aus ihnen geht hervor, daß nur Schwefel- und Salzsäure als Fällungsmittel in Betracht kommen, daß aber die erstere Säure genauer und schärfer einwirkt. Alle anderen Säuren kommen nicht in Betracht.

\section{Versuche, die Reinheit dergewogenen Säure festzustellen.}

Da man bei den verschiedenen Bestimmungen verschieden reine Säuren beziehungsweise Salze zur Wägung bringt, genügt es nicht, bei einer Kritik der Glycyrrhizinsäurebestimmungen die erhaltenen und gewogenen Mengen miteinander zu vergleichen. Es muß auch Rücksicht genommen werden auf die Reinheit derselben. Die einzige Prüfung, die das zu tun versucht, ist die $\mathrm{H}$ a $f \mathbf{f} \mathrm{n}$ er's. Er bildet das Baryumsalz, bestimmt durch Abrauchen mit Schwefelsäure den Baryumgehalt, indem er das gebildete Baryumsulfat wägt, und hat damit eine Möglichkeit, die Reinheit der Glycyrrhizinsäure festzustellen. Wenn ich nun versuchen würde, in der vorliegenden Arbeit den Reinheitsgrad der Säure festzustellen, so könnte ich naturgemäß nicht ohne weiteres dem $H$ aff $n$ er'schen Vorschlage folgen. H af $\mathbf{n}$ er's Acetonauszüge bezwecken eine Rei- 
nigung der Säure, die ja garnicht in meiner Absicht liegt und hier direkt falsch wäre. Ich versuchte die verschiedensten Aenderungen der $\mathrm{H}$ a f f $\mathrm{n}$ e r'schen Vorschrift, ohne aber zum Ziel zu gelangen. Vorerst möchte ich hier einige von mir hei dieser Gelegenheit festgestellte Tatsachen bringen. Die filtrierte Ammonglycyrrhinatlösung gibt, eingedampft, bei $100^{\circ}$ getrocknet, dann wieder in Wasser gelöst, einen unlöslichen Rückstand. Das bei $100^{\circ}$ getrocknete Ammonglycyrrhinat, wie es gewogen worden ist, kann, ohne daß eine Reinigung eingetreten ist, nicht in das Baryumsalz übergeführt werden. Die bei $100^{\circ}$ getrocknete Glycyrrhizinsäure ist in 95 a. $\mathrm{H}$. Alkohol nicht wieder ohne Rückstand in Lösung zu bringen. Auch die Glycyrrhizinsäure selbst kann ohne Reinigung nicht in das Baryumsalz übergeführt werden. In dem erhaltenen Baryumsalz könnte der Baryumgehalt festgestellt und damit ein Rückschluß auf die Reinheit des Salzes getan werden. Dies berechtigt mich aber nicht, auch nur einen annähernden Schluß auf die Reinheit bezièhungsweise Unreinheit der gewogenen Säure beziehungsweise des Ammonsalzes zu ziehen. $\mathrm{Zu}$ allen diesen angeführten Tatsachen und Bedenken kommen dann noch die gegen die Reinheitsprüfung $H$ a f $\mathrm{n}$ e r's vorgebrachten Einwände.

Ich komme also zu dem Schluß, daß nach dem Vorschlage H a f f ner's und auch sonst durch andere Bestimmungen es nicht möglich ist, den Reinheitsgrad der Säure auch nur annähernd festzustellen. Die von $\mathrm{H}$ a f $\mathrm{f} \mathrm{e} r$ in seinen Aufstellungen gebrachten Reinheitsgrade der verschiedenen Säuren kann ich nicht anerkennen. Nach seinen eigenen Angaben kann er sie kaum erhalten haben - und wenn doch, so geben sie kein richtiges Bild - und einen anderen Weg gibt er nicht an. So wünschenswert und für eine abschließende Bewertung der einzelnen Prüfungsvorschläge unerläßlich auch eine solche genaue Feststellung des Reinheitsgrades sein möge, ist man hier doch nur auf Geschmack und Aussehen als Prüfungsmaßstab angewiesen. Und hierbei zeigt es sich dann, daß der ammoniakalische Auszug zweifellos eine unreinere Säure gibt, als der wässerige, und daß aus demselhen Grunde die Verwendung von Spiritus einem rein wässerigen Auszug vorzuziehen ist. Durch das Aeußere der zur Wägung gebrachten Säure zeigt sich auch schon, daß die Reinigung derselben mit Alkohol nach $D$ i e hl eine bedeutende ist. Das nach $\mathrm{D}$ i e h l's Vorschrift gewonnene Ammonglycyrrhinat ist von hellbrauner Farbe, während die nach anderen Bestimmungen erhaltenen Salze schwarzbraun gefärbt sind.

(Fortsetzung folgt.) 